\title{
Review of: "Characterisation of circulating tumour cell phenotypes identifies a partial-EMT sub- population for clinical stratification of pancreatic cancer"
}

Bo Deng ${ }^{1}$

1 3rd Military Medical University

Potential competing interests: The author(s) declared that no potential competing interests exist.

1. Healthy individuals and cases with benign tumors or pancreatitis should be included as controls.

2. Other methods for CTCs detection should be used for validation 